\title{
Application Research of the Non-Destructive Testing Using Optical Fiber Sensor
}

\author{
Zhiyong Dai, Hong Zhang, Hongliang Wu, Baoji Li, Zengshou Peng \\ School of Optoelectronic Science and Engineering, University of Electronic Science and Technology of China, Chengdu, China \\ Email: emzydai@uestc.edu.cn
}

How to cite this paper: Dai, Z.Y., Zhang, H., Wu, H.L., Li, B.J. and Peng, Z.S. (2019) Application Research of the Non-Destructive Testing Using Optical Fiber Sensor. Journal of Applied Mathematics and Physics, 7, 2917-2925.

https://doi.org/10.4236/jamp.2019.711201

Received: November 15, 2019

Accepted: November 24, 2019

Published: November 27, 2019

\begin{abstract}
In this paper, an optical fiber sensor is designed by using optical Faraday effect. It is composed of fiber collimator, polarizer, magneto-optical crystal and mirror. Based on the magnetic flux leakage (MFL) theory, The optical fiber sensor was placed between two permanent magnets with the N-pole. Therefore, the optical fiber sensing system was built to detect the defective ferromagnetic objects. Theoretical and experimental studies shown that the system can identify a little defects, such as irons' blind hole (diameter $\varphi=3 \mathrm{~mm}$, depth $t=4 \mathrm{~mm}$ ), irons' grooves (length $\ell=30 \mathrm{~mm}$, width $\omega=10 \mathrm{~mm}$ ), hole $(\varphi=3 \mathrm{~mm})$ and crackle etc. The system has the characteristics of small size, high sensitivity, fast signal response and high resolution. In terms of the defective oil and gas pipelines detection, The optical fiber sensing system is used in non-destructive testing, which will be valuable and meaningful.
\end{abstract}

\section{Keywords}

Optical Fiber Sensor, High Sensitivity, Non-Destructive Testing

\section{Introduction}

In the field of non-destructive testing, especially in oil and gas pipelines, there are five commonly used non-destructive testing technologies: Radiographic Testing (RT), Ultrasonic Testing (UT), Magnetic powder Testing (MT), Penetration Testing (PT), Eddy Current Testing (ECT). Certainly, they have their own advantages and disadvantages. At present, ultrasonic testing (UT) technology [1] and Magnetic Flux Leakage Testing (MFLT) technology [2] are the most mature and widely used in pipeline and plate inspection. However, there are some shortcomings in ultrasonic testing technology, such as the need for coupling agent and the high requirement for the pipe surface cleanliness. For magnetic flux leakage testing (MFLT), it has some problems such as the low sensitivity of 
the sensor used and the large instrument. Recently, the electromagnetic eddy current testing (ECT) method [3] has been rapidly developed in the field of internal detection, due to its non-contact detection, test instrument assembly and simple operation. However, because the eddy current method relies on the skin effect of metal conductor, the defect depth of objects and the interference of electromagnetic wave will bring difficulties to the signal identification.

According to the theory of magnetic flux leakage on the metal surface, when the ferromagnetic object is magnetized, the magnetic field line on the object surface will be distorted at the defect, and a part of the magnetic field line will form a magnetic field leakage through the inner and outer surface of the object. The defect information of the object can be obtained by detecting the strength of the leakage magnetic field. According to the report of using optical method for magnetic leakage detection, there are optical interference method [4], photonic crystal fiber sensor method [5], fiber Bragg grating (FBG) sensor method [6] etc. However, these optical methods have the disadvantages that the demodulation system is complicated and bulky, the sensor is difficult to manufacture, and the signal is affected by temperature. In this paper, the optical magneto-optical effect is used. When the input light passes through the magnet-optical crystal, the direction of its light vector will be deflected due to the change of the external magnetic field, and after passing through a linear polarizer, the light intensity of its output will be changed. Consequently, the purpose of measuring the weak magnetic fields can be achieved by detecting the output light intensity. Because the optical signal has no electromagnetic interference, no temperature interference, and fast response, this method has great advantages in the field of non-destructive testing (NDT).

\section{Fiber Sensor Design by Optical Faraday Effect}

As shown in Figure 1, the fiber sensor consists of four parts, a fiber collimator, a linear polarizer, a magneto-optical crystal, and a mirror. The operating principle of the sensor can be described as: The linearly polarized light is generated after the incident light passes through the collimator and linear polarizer. This beam transmitted the magneto-optical crystal, its light vector will rotate an angle $(\theta)$ that is proportional to the magnetic induction intensity and the propagation distance (I). Finally, the rotating light is returned through the mirror and recoupled into the fiber to generate the output light. The detailed process can be expressed by mathematical formulas.

$$
\theta=V B l
$$

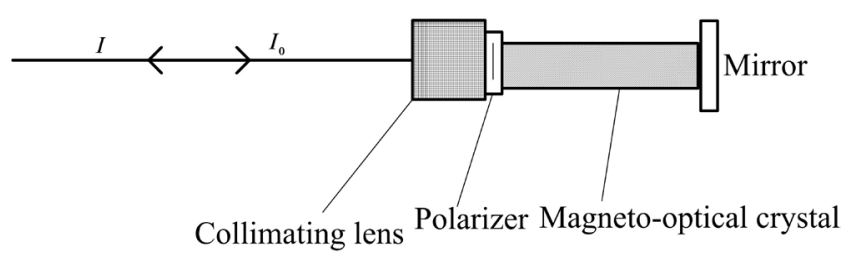

Figure 1. Schematic diagram of optical magnetic field sensor. 
In formula (1), $V$ is called Verdet constant, which is related to light wavelength. When the linearly polarized light passes through the magneto-optical crystal in the magnetic field, the vibration surface will rotate, if the magnetization intensity is not saturated, the rotation angle $(\theta)$ of the vibration surface is directly proportional to the magnetization intensity $M$ and the passing distance $(I)$, that could be written as:

$$
\theta=F \frac{M}{M_{0}} l
$$

In the above formula (2), $M_{0}$ is saturation magnetization, $F$ is called Faraday optical rotation coefficient. Furthermore, the linearly polarized light passes through the crystal with strong rotation ability, and its light vector deflects. The light intensity obtained through the output of the polarizer is:

$$
I=I_{0} \cos ^{2} \alpha
$$

In formula (3), $\alpha=2 \theta$. Which means the light vector is rotated twice. $I$ is represented the output light intensity and $I_{0}$ is the incident light intensity. The light intensity is the largest when the incident light is parallel $\left(\theta=0^{\circ}\right)$ to the optical axis of the reflected light, so the change of the external magnetic field can be measured by detecting the change of the light intensity in the experiment.

\section{Research on Numerical Calculation}

From the above, it can be known that the optical signal comes from the change of external weak magnetic field. So firstly in this section, the leakage magnetic field on the metal surface is analyzed by numerical simulation.

\subsection{Theoretical Calculation of Leakage Magnetic Field}

As shown in Figure 2(a), the ferromagnetic sample is placed in the magnetic field, the cross-sectional area of the sample without defect is $S_{1}$, the cross-sectional area of the sample with defect is $S_{2}$. Obviously, $S_{1}>S_{2}$. As shown in Figure 2(b), according to the magnetic resistance $R=l / \mu S$, the magnetic resistance $R_{2}$ at $S_{1}$ is greater than that $R_{1}$ at $S_{2}$, namely $R_{2}>R_{1}$. Because of the principle of magnetic continuity, if the flux passes through $S_{2}$, it will be divided to form a leakage flux $\Phi_{l}$.

The formation of leakage magnetic field can be understood as: because the

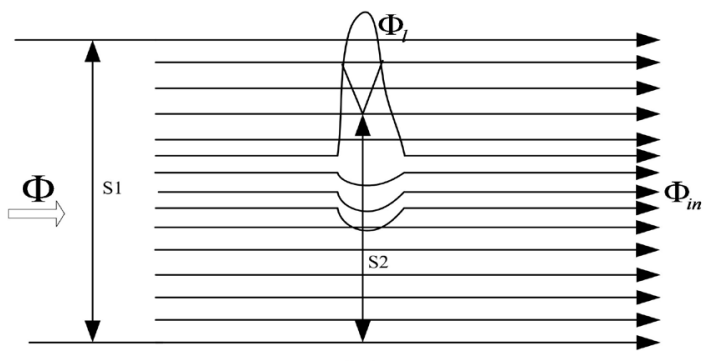

(a)

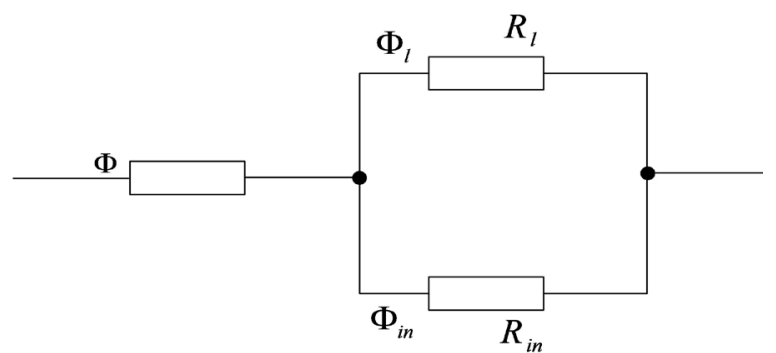

(b)

Figure 2. Formation of leakage magnetic field. (a) Metal leakage field; (b) Formation principle. 
permeability of air is far lower than the ferromagnetic material, if there is discontinuity or crack in the ferromagnetic material, the magnetic induction line will pass the irons with high permeability preferentially, which forces part of the magnetic induction line to bypass the defect and form the compression of the magnetic induction line. However, the number of magnetic induction lines that can be accommodated in the continuous part of the irons is limited. Because the same-sex magnetic induction lines are mutually exclusive, some of the magnetic induction lines pass through from the discontinuity. The other part of the magnetic induction lines follow the refraction law and enter the air almost vertically from the irons' surface. After passing through the defects, they return to the irons to form a magnetic leakage field.

The essence of leakage magnetic field is the distortion of magnetic field caused by defects. It is difficult to directly solve the leakage magnetic field intensity $\mathbf{B}$ according to the equations of Maxwell's equations. However, the numerical finite element method is commonly used for calculation.

\subsection{The Finite Element Calculation}

In order to verify the feasibility of detecting metal surface defects by optical weak magnetic field detection technology, this paper uses the Magnetic-nodal module of ANSYS software to calculate the leakage magnetic field of the testing irons with corrosion defects. In the simulation, the two-dimensional geometric model of irons is established, including testing irons with defect, permanent magnet, steel brush and yoke. The specific parameters are shown in Table 1 below, and the geometric model is shown in Figure 3.

The material attributes of each part are assigned according to the solid geometry model. In Figure 3(a), A1, A6, A8 and A9 are air with permeability of 1; $\mathrm{A} 2$ is $\mathrm{N}$ pole of permanent magnet, $\mathrm{A} 4$ is S pole of permanent magnet; $\mathrm{A} 3$ and A5 are slider; A7 is yoke iron and A10 is testing iron plate. The plate's corresponding magnetic characteristics is shown in Figure 3(b).

\subsection{Calculation Results}

By using the ANSYS software, the electric magnetic field is analyzed according the model parameters in Table 1 and solid geometry model as shown Figure 3.

Table 1. Parameters of solid 2D geometric model.

\begin{tabular}{ccccc}
\hline \multirow{2}{*}{ Order } & \multicolumn{4}{c}{ 2D geometry model parameters } \\
\cline { 2 - 5 } & Name & Thickness $(\mathrm{cm})$ & Length $(\mathrm{cm})$ & Permeability \\
\hline 1 & Yoke iron & 2 & 20 & 186,000 \\
3 & Permanent-magnet & 1 & 2 & 1.05 \\
4 & Slider & 0.5 & 2 & 0.99 \\
5 & Ferromagnetic plate & 0.8 & 40 & B-H \\
& Defect & 0.4 & 1 & 1 \\
\hline
\end{tabular}


The two magnets with $\mathrm{N}$ poles are placed, their distance is $20 \mathrm{~cm}$. The magnetic force line distribution map is showed in Figure 4. Figure 4(a) describes the distribution of the flux lines of the defective plate, and (b) depicts the two-dimensional flux distribution of the perfect Ferromagnetic plate. The density of the flux lines represents the strength of magnetic field.

It can be seen that the magnetic intensity in the magnet and the yoke iron is very strong and reaches the magnetic saturation. But the surrounding air also produces a relatively weak magnetic field. Compared the two pictures in Figure 4, it is found that there is a tiny difference of magnetic field at defect location. The magnetic flux lines in Figure 4(a) is a little distorted, while Figure 4(b) reveals that the lines is distributed homogeneously. According to the Faraday Effect, when the magneto-optical crystal does not reach magnetic saturation, it has extremely high sensitivity to detect the weak magnetic field variation caused by the defect. But if the magnetic field is invariable, the light vector of the incident light is constant so that the optical fiber sensor doesn't response to the magnetic field.

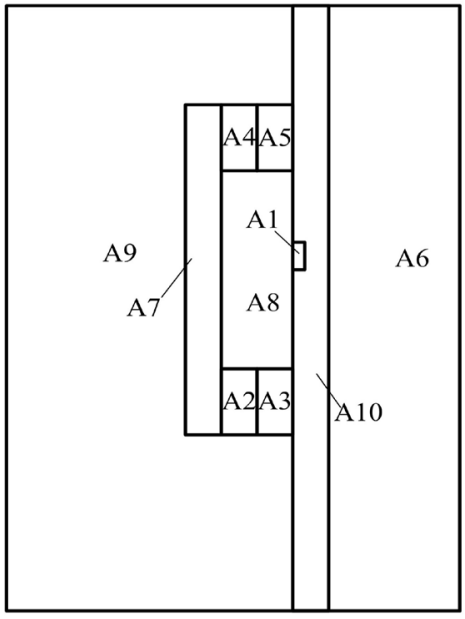

(a)

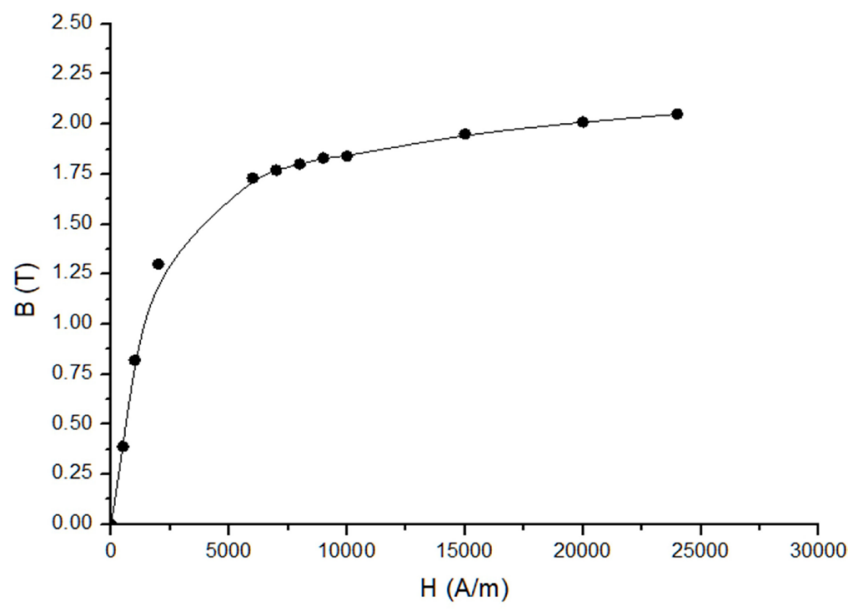

(b)

Figure 3. 2D model. (a) Geometry model; (b) Magnetic characteristic of ferromagnetic plate.

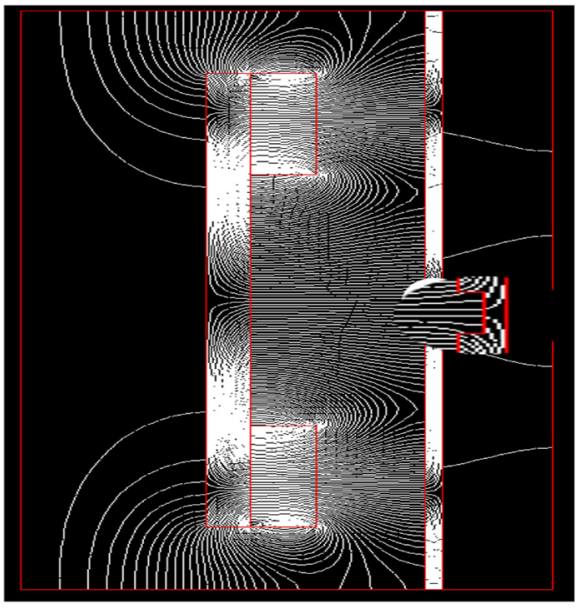

(a)

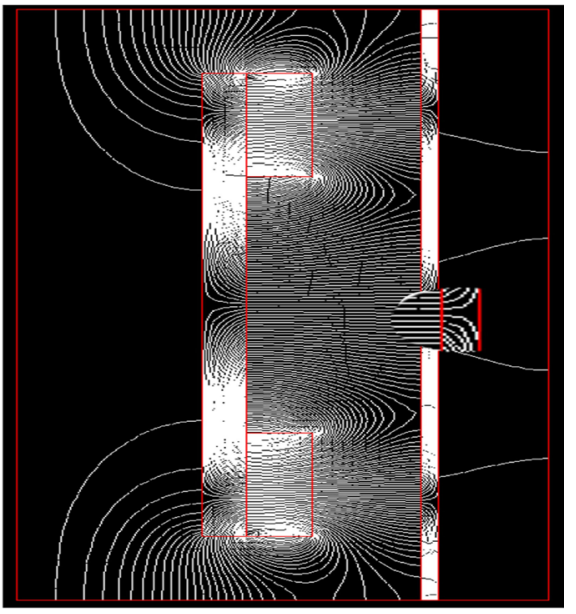

(b)

Figure 4. Distribution of 2D flux lines. (a) Testing iron with defect; (b) No defect. 
Furthermore, we compare the magnitude of leakage magnetic field at the same position in the air for the ferromagnetic plates' surface with defect or not. According to the finite element decomposition method, the magnetic induction $\mathrm{B}$ is decomposed into the $\mathrm{X}$ direction $(\mathrm{Bx})$ and the $\mathrm{y}$ direction (By). The $\mathrm{X}$ direction indicates that the magnetic line is perpendicular to the direction of propagation of the light in the crystal, otherwise the $\mathrm{Y}$ direction indicates that the direction of the magnetic line is parallel to the direction of light propagation. Naturally, the value of By does not affect the intensity of light.

As a result, the magnetic induction intensity (the $\mathrm{Bx}$ and $\mathrm{By}$ ) distribution curve is shown in Figure 5. The height of the sensor from plate is $1 \mathrm{~mm}$. In Figure 5(a), the peak value of the magnetic induction intensity in the $x$ direction is about $17(\mathrm{mT})$. The theoretical calculation and analysis indicate that the change of the magnetic field will cause optical Faraday Effect of light.

Therefore, the optical sensor can sense the distorted leakage magnetic field. The optical weak magnetic field detection technology has the characteristics of high response speed and high sensitivity.

\section{Experimental Research}

\subsection{Experimental Setup}

In this section, the results of theoretical simulation are verified by experiments. Firstly, an optical test system is built to detect the change of light intensity, and the schematic diagram of the system is shown in Figure 6 . The wavelength of the light is $\lambda=1550(\mathrm{~nm})$. The optical power is $2(\mathrm{mw})$. The received optical power is $10(\mu \mathrm{w})$ without magnetic field.

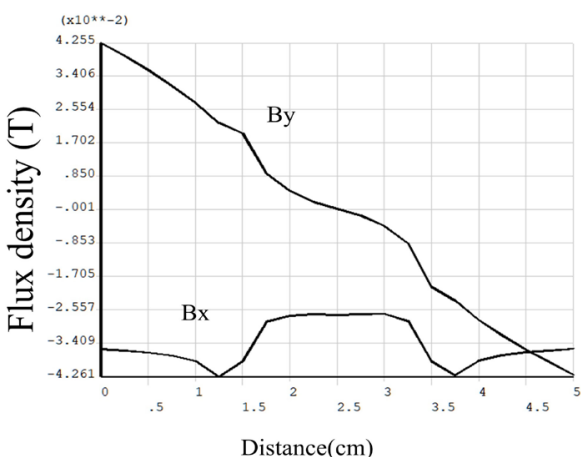

(a)

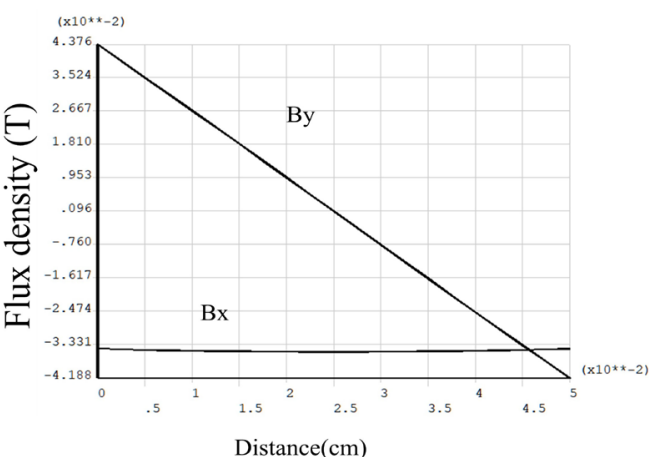

(b)

Figure 5. The Mag flux dens. (a)Testing iron with defect; (b) No defect.

Testing metal

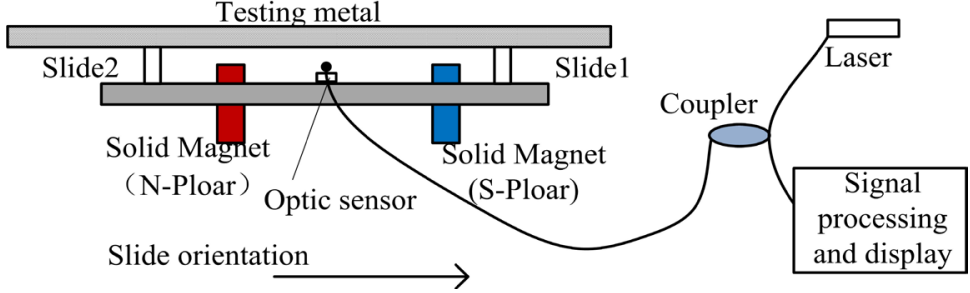

Figure 6. Optical leakage magnetic field detection system. 
Secondly, choose the ferromagnetic plate with corrosion. The size of the plate is $300^{\star} 500^{\star} 10\left(\mathrm{~mm}^{3}\right)$. The defect depth is $60 \%, 40 \%$ and $20 \%$ of the thickness respectively (thickness $t=10(\mathrm{~mm}))$. The shape of corrosion is circle $(\varphi=10(\mathrm{~mm}))$ and groove (length $l=30(\mathrm{~mm})$, width $w=10(\mathrm{~mm})$ ).

Circular rubidium iron boron magnet was used in the experiment, its outer diameter is $18(\mathrm{~mm})$, inner diameter is $4(\mathrm{~mm})$ and thickness is $3(\mathrm{~mm})$. The magnets are placed with $\mathrm{N}-\mathrm{N}$ at the distance of $10(\mathrm{~cm})$, the optical sensor was placed in the middle, the distance between the sensor and the plate surface is 3 $(\mathrm{mm})$. Similarly, the distance between the magnet and the plate surface is $3 \mathrm{~mm}$. Fix the optical sensor, magnet and yoke. Slide them over the iron plate's surface at a constant speed. The magnetic flux leakage signal was picked up by the optical weak magnetic field sensor. After magneto-optical and photoelectric conversion, the change of light intensity can be observed on the display.

\subsection{Experimental Results}

The optical sensor passes through the circular blind hole 1, 2 and 3 from left to right on a uniform speed. Because the depth of the three kinds of blind holes was the thickness' 60\%, 40\% and 20\% respectively (as shown in Figure 7(a)). The leakage flux from the plate into the air is varies. it can be seen (as shown in Figure $7(b))$ that the optical magnetic sensor passes through the No. 1 circular blind hole $\left(\varphi=6 \mathrm{~mm}\right.$ ), the optical signal intensity $\Delta v_{1} \approx 300(\mathrm{mv})$, the light signal intensity of No. 2 blind hole was $\Delta v_{2} \approx 250(\mathrm{mv})$, and the signal intensity of No. 3 blind hole was $\Delta v_{3} \approx 210(\mathrm{mv})$.

The same experimental method was used for the circumferential groove, and the results were shown in Figure 8. When the corrosion depth of No. 1 groove was $2 \mathrm{~mm}(20 \% * t=2)$, corresponding signal strength was $\Delta v_{1}=200 \mathrm{mw}$. When

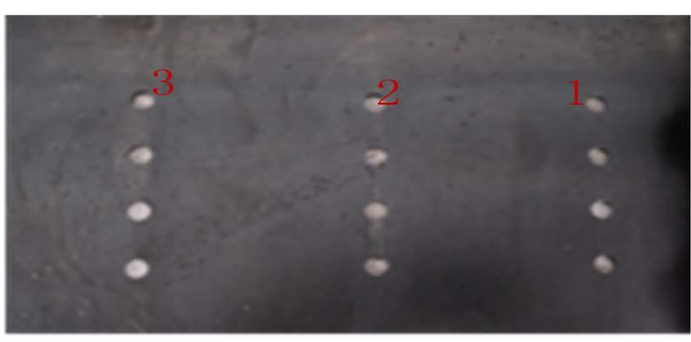

(a)

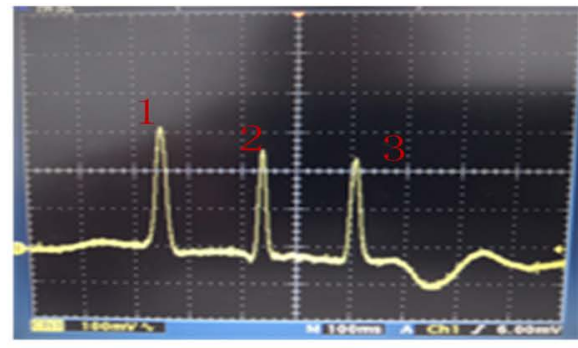

(b)

Figure 7. The testing result of the circle defective iron plate. (a) The plate; (b) The signal.

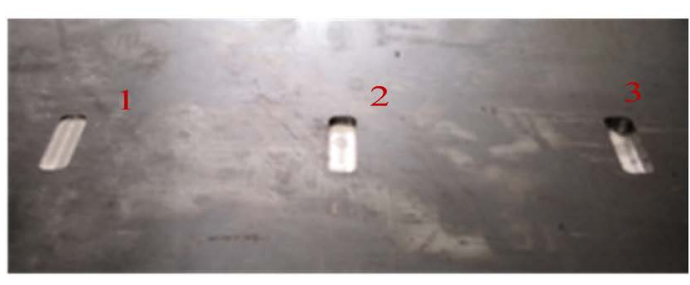

(a)

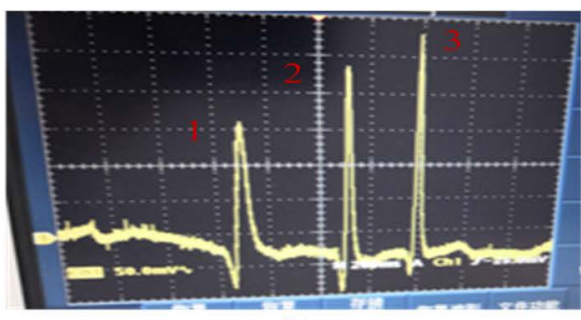

(b)

Figure 8. The testing result of the groove defective iron plate. (a) The plate; (b) The signal. 


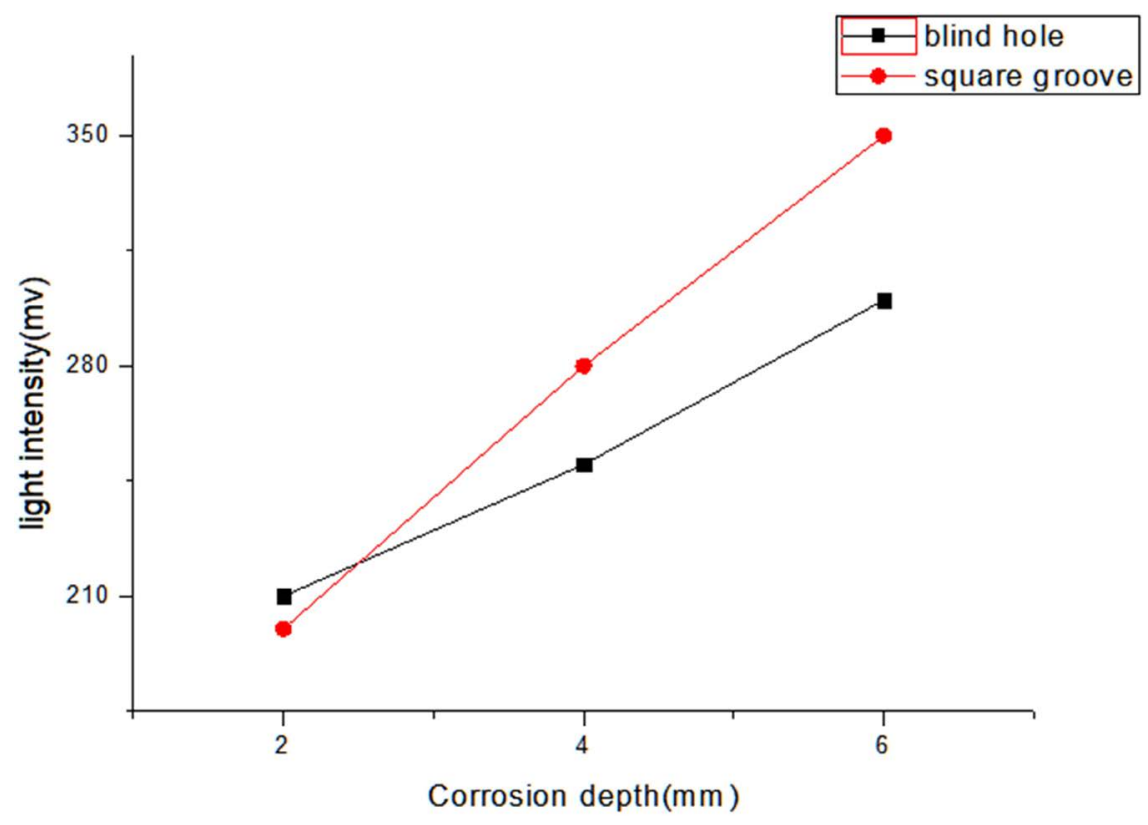

Figure 9. The relationship between the depth of corrosion and the optical signal intensity.

the corrosion depth of No. 2 groove was $4 \mathrm{~mm}(40 \% * t=4)$, corresponding signal strength was $\Delta v_{1}=280 \mathrm{mw}$. When the corrosion depth of No 3 groove was $6 \mathrm{~mm}(60 \% * t=6)$, corresponding signal strength is $\Delta v_{1}=350 \mathrm{mw}$.

Figure 9 above depicts the relationship between the corrosion depth and optical signal intensity. The curve shown that the depth of corrosion on the ferromagnetic plate's surface is positively related to the intensity of light. And the gradient of the signal of the circular blind hole is less than that of the long groove because of the optical sensor scanning the surface of the plate in one dimension. Besides, the signal intensity may be related to the corrosion shape.

\subsection{Discussion the Experimental Results}

Based on the above experimental results, if the minimum signal strength that can be recognize is $50 \mathrm{mv}$, The fiber sensor can detect the diameter of the hole is about $3 \mathrm{~mm}$, and the corrosion depth is $5 \%^{\star} t$ at least. However in order to correctly obtain the spatial physical information of the defect, it is necessary to control the movement speed of the sensor and increase the number of sensors.

\section{Conclusion}

Through the theoretical and experimental analysis of the leakage magnetic field on the ferromagnetic plate, it was proved that the optical fiber sensing system can correctly detect the defects on the metal surface. At least, the fiber sensor could detect the diameter of the hole is about $3 \mathrm{~mm}$, and the corrosion depth is $5 \%{ }^{\star}$ t. More than that, the magnitude of the fiber sensor's sensitivity reached 1 $\mathrm{mT}$. In the future, we will study the operation of array sensors to enhance the resolution of defects. This research has a great significance in nondestructive testing (NDT) of oil and gas pipelines. 


\section{Acknowledgements}

This project was supported by The National Basic Research Program of China (No. 2016ZX05021-005-010HZ).

\section{Conflicts of Interest}

The authors declare no conflicts of interest regarding the publication of this paper.

\section{References}

[1] Alobaidi, W.M., Kintner, C.E. and Alkuam, E.A. (2017) Experimental Evaluation of Novel Hybrid Microwave/Ultrasonic Technique to Locate and Characterize Pipe Wall Thinning. Journal of Pressure Vessel Technology, 2017, Article ID: 011501. https://doi.org/10.1115/1.4038517

[2] Liu, B., He, L.Y. and Zhang, H. (2017) The Axial Crack Testing Model for Long Distance Oil-Gas Pipline Based on Magnetic Flux Leakage Internal Inspection Method. Measurement, 103, 275-272. https://doi.org/10.1016/j.measurement.2017.02.051

[3] Luo, Q.W. (2018) Research on the Defect Detecting Methods of Ferromagnetic Pipes Based on Electromagnetic Eddy Current. M. A. University of Electronic Science and Technology of China, Chengdu.

[4] Shen, T., Sun, B.C. and Feng, Y. (2018) Mach-Zehneder Interference All-Fiber Sensor for Measurement of Magnetic Field and Temperature. Optics and Precision Engineering, 1338-1344. https://doi.org/10.3788/OPE.20182606.1338

[5] Li, J.H., Pei, L., Wang, J.S., Wu, L.Y., Ning, T.G. and Zheng, J.J. (2019) Chinese Journal of Lasers, 2019, Article ID: 0210002.

[6] Wang, J.G., Zeng, J., Li, Z.H., Guo, X.H., Gong, X.J., et al. (2019) Research on Fiber Magnetic Field Sensor Based on LPG and FBG Composite Structure. SPIE, 2019, Article ID: 106180B-1. 